\title{
Extracción de diente ectópico en comunicación con piso de cavidad nasal: reporte de caso
}

\author{
Extraction of an ectopic tooth in communication with a nasal cavity floor: $A$ \\ case report
}

\author{
Andrea del Pilar Albarracín-García ${ }^{1}$, Luisa Fernanda Muñoz ${ }^{2}$, Gerardo Enrique Albarracín-García ${ }^{3}$
}

Citación: Albarracín-García AP, Muñoz LF, Albarracín-García G. Extracción de diente ectópico en comunicación con piso de cavidad nasal: reporte de caso. Ustasalud 2020;19. 35-41

Licencia Creative Commons

(ㄷ) (1) (9) $\Theta$ alterarlos, adicionalmente se debe reconocer la autoría de las personas que figuran en las publicaciones, pero estas no podrán ser comercializadas.

\section{Resumen}

La erupción ectópica es una ocurrencia poco evidenciada en la consulta odontológica. Esta anomalía generalmente es asintomática y suele únicamente descubrirse mediante radiografías en una revisión dental rutinaria, sin embargo, se puede ver acompañada de procesos patológicos, como quistes, reabsorción ósea o infección, entre otros. El objetivo de este texto es presentar el manejo clínico y tratamiento de un diente maxilar en posición ectópica en comunicación con la fosa nasal derecha. Se reporta el caso clínico de una mujer de 45 años de edad, con síntomas de dolor en el paladar y antecedentes de epistaxis. Al examen clínico presenta una lesión en el paladar, de consistencia blanda y fluctuante con leve drenaje de material purulento. Se descubre la presencia de un diente ectópico mediante una cirugía exploratoria y se realiza la exodoncia de este. Se hace seguimiento constante durante cuatro meses y la paciente presenta una recuperación satisfactoria, refiriendo alivio total de sintomatología; todo esto a pesar de las limitaciones generadas por el nivel socioeconómico del paciente y el entorno en el cual se lleva a cabo la atención odontológica. Lo anterior, evidencia la vital importancia del correcto diagnóstico de anomalías dentales, para poder brindar un tratamiento adecuado y oportuno.

Palabras clave: Erupción ectópica de dientes, diente no erupcionado, absceso, epistaxis, cavidad nasal.

\begin{abstract}
Ectopic tooth eruption is a rare occurrence to witness during a dental consultation. This anomaly is generally asymptomatic and frequently discovered only by radiographs taken during a routine dental check-up, however, it can be followed by pathological processes such as cysts, bone resorption, or infection, among others. The aim of this text is to present the clinical management and treatment of a maxillary tooth in an ectopic position in communication with the right nasal cavity. This is a report of the clinical case of a 45-year-old female patient who seeks dental attention during a health brigade, located in a rural place in Colombia, reporting symptoms of pain on the roof of the mouth and a history of epistaxis. Upon clinical examination, she presented a soft, fluctuating lesion on the palate with slight drainage of purulent material. The presence of an ectopic tooth is discovered through exploratory surgery, and the tooth is extracted. Constant follow-up is carried out for four months, and the patient shows a satisfactory recovery, reporting total relief of symptoms; all this despite the limitations generated by the socioeconomic level of the patient and the environment in which the dental care is carried out. Correctly diagnosing dental anomalies is of utmost importance in order to provide an adequate and timely treatment.
\end{abstract}

Keywords: Ectopic tooth eruptions, unerupted tooth, abscess, epistaxis, nasal cavity.
1 Odontóloga, Universidad Santo Tomás, Bucaramanga, Colombia 2 Estudiante de $X$ semestre, Facultad de Odontología, Universidad Santo Tomás, Bucaramanga, Colombia.

3 Especialista en Endodoncia, Universidad Santo Tomás, Bucaramanga, Colombia.

Autor de correspondencia: Andrea del Pilar Albarracín-García. Correo electrónico:

andrea_albarracin15@hotmail.com
Recibido para publicación: 25 de julio de 2020

Aceptado para publicación:

9 de octubre de 2020 


\section{INTRODUCCIÓN}

Las anomalías que pueden suceder en el proceso de erupción normal de los dientes implican cambios en la formación dental, afectando el evento conocido como odontogénesis. Este incluye varios procesos fisiológicos de crecimiento dental, formación y cambios morfológicos, los cuales se dividen en dos fases: morfogénesis e histogénesis. Normalmente existe una armonía entre el crecimiento óseo de los maxilares y el desarrollo dental, pero un desbalance provocado por disturbios endógenos o exógenos como factores congénitos o ambientales, pueden provocar alteraciones dentro de estos procesos y la aparición de anomalías como dientes en posición ectópica o dientes supernumerarios, los cuales tienen ciertas diferencias ${ }^{1,2}$. Un diente supernumerario es un diente extra o adicional a la cantidad de dientes que deben estar presentes en cada hemiarcada (8 dientes), mientras que un diente ectópico, es un diente que se encuentra fuera de su posición normal, es decir que no se aloja dentro del hueso alveolar ${ }^{2}$. Algunos factores que influyen para que un diente supernumerario se convierta en un diente ectópico, son: el apiñamiento, desplazamiento dental, erupción retardada, diente impactado y presencia de quistes; lo cual, como consecuencia, termina desencadenando un trastorno en la oclusión ${ }^{1}$. El problema es que, en muchos de estos casos, por temor o desconocimiento no se da un tratamiento oportuno e ideal.

Un diente ectópico es sobre todo un diente que se encuentra en una posición inusual o diferente a su posición anatómica habitual. Estos dientes generalmente se pueden encontrar en zonas sub condilares, en la rama mandibular, en el seno maxilar, zona infraorbitaria, paladar, fosas nasales o en la sínfisis mandibular ${ }^{3}$. Todo diente que se encuentre ajeno a su ubicación anatómica normal que es el límite del hueso alveolar, se considera un diente en posición ectópica ${ }^{4}$.

Estudios previos reportan que este tipo de casos se presenta en un $0,5 \%$ a un $0,9 \%$ de la población, con mayor prevalencia en el sexo masculino y suelen presentarse debido a su dirección eruptiva anormal y posición final fuera de los límites anatómicos ${ }^{4}$. Algunas de las causas frecuentes de los dientes ectópicos en la región nasal, suelen ser pacientes que padecen de labio y paladar hendido lo cual afecta la erupción ideal dentro de los maxilares, el desplazamiento dental causado por un trauma, desplazamiento iatrogénico del órgano dental, desarrollo de una infección odontogénica, obstrucciones en el patrón de erupción, herencia, insuficiente crecimiento maxilar, tumores, quistes, tratamientos ortodónticos inadecuados o apiñamiento dental ${ }^{5,6}$. Cuando estos dientes aparecen en una posición anormal, como por ejemplo, en la lengua, orofaringe, ovarios, testículos y se encuentra acompañado de otros tejidos diferentes al tejido dental, se conoce con el nombre de teratoma ${ }^{4}$.

Estos dientes suelen ser asintomáticos en la mayoría de los casos, pero pueden dar paso a la aparición de otras patologías como son los quistes odontogénicos, reabsorción ósea, infección, obliteración pulpar, incluso pueden llegar a producir dolor neurálgico. En los casos asintomáticos, estos dientes solo son diagnosticados cuando se descubren mediante radiografías rutinarias y el paciente desconoce por completo su caso ${ }^{7}$.

El objetivo del presente reporte de caso es mostrar el manejo odontológico de un diente en posición ectópica ubicado en el paladar, en comunicación con la cavidad nasal con el fin de aportar mayor conocimiento y técnicas opcionales para el abordaje y tratamiento de casos similares.

\section{REPORTE DE CASO}

Paciente de sexo femenino de 45 años de edad, no reporta compromiso sistémico o consumo de fármacos. Reporta dolor de un resto radicular superior, molestia en el paladar y sangrado nasal esporádico por la fosa nasal derecha.

En el examen clínico se observa un resto radicular del primer molar superior izquierdo permanente con sintomatología dolorosa, y lesión en zona derecha del paladar, aproximadamente $25 \mathrm{~mm}$ de diámetro, de consistencia blanda y fluctuante (Figuras 1a y 1b).

La paciente procede a firmar un consentimiento informado legal para la exodoncia del resto radicular y el drenaje de la lesión, luego de explicar los riesgos y beneficios del procedimiento. Se hace la exodoncia 
del resto radicular y al finalizar el procedimiento se realiza interconsulta médica por el sangrado nasal esporádico que la paciente refiere. El médico valora a la paciente y decide enviarle profilaxis antibiótica con Diclofenaco para proceder al drenaje de la lesión. La paciente, después de su revisión médica, regresa al área de odontología para iniciar el procedimiento de drenaje. Se bloquea el nervio palatino mayor, dentario medio y con un bisturí se hace una incisión vertical sobre la lesión, pero se observa leve material purulento. Se explora la zona con una pinza Kelly curva y se le proponen dos opciones a la paciente: a) suturar la incisión actual y dejarla en observación, tal cual como ya durante dos años venía siendo tratada con procedimientos similares y sin tener ningún éxito, o b) firmar un consentimiento informado legal adicional para realizar una cirugía exploratoria asumiendo el riesgo de posibles complicaciones que dicho procedimiento podría presentar. La paciente opta por la cirugía exploratoria y firma el consentimiento informado legal.

Se complementa la incisión previa, con otra intrasurcular por la zona mesiopalatina (M-P) del diente 14, terminando con una relajante vestíbulodistal (VD) y se levanta el colgajo mucoperióstico, logrando así una mayor visibilidad del campo operatorio. Se lava la zona con solución salina y se aplica solución hemostática con principio activo de sulfato férrico, para permitir una mejor inspección del campo de trabajo. Se realiza la exploración de la zona con ayuda de un periostótomo de Molt y se palpa dentro de la lesión, encontrando un tejido duro y denso, de color blanquecino, compatible con un órgano dental (Figuras $2 \mathrm{a}, 2 \mathrm{~b}$ y $2 \mathrm{c}$ ). Una vez localizado el diente retenido, con ayuda de un elevador recto acanalado delgado se hace una luxación en sentido vertical y, posteriormente, se procede a realizar prehensión, utilizando el fórceps No.65, pero al hacer los movimientos de tracción del diente, la paciente reporta dolor en la zona alta del paladar; por lo cual se procede a aplicar una técnica anestésica local, para el bloqueo regional del nervio infraorbitario (Figuras $2 \mathrm{~d}$ y $2 \mathrm{e}$ ).

Se esperan dos minutos para que el anestésico haga su efecto y con movimientos de leve lateralidad, rotación y tracción, se logra la extracción del diente. El órgano dental extraído tiene ausencia de corona dental, presencia de una sola raíz y la entrada del conducto pulpar desde la porción cervical hasta la zona apical se observa de color grisáceo, compatible con necrosis pulpar, causa posible de la lesión y proceso infeccioso de la paciente (Figura 2f).

Se realiza curetaje de la zona con el fin de eliminar todo el tejido granulomatoso presente y promover la formación del coágulo, posteriormente se verifica que no exista presencia de espículas y se lava con solución salina, desinfectando adicionalmente con clorhexidina y finalmente se sutura en " $X$ " en la incisión palatina y punto simple en la incisión relajante (Figura $2 \mathrm{~g}$ ). Se dan indicaciones de cuidado post quirúrgico y se complementa la terapia farmacológica con Naproxeno en tabletas de $500 \mathrm{mg}$ por vía oral cada 6 horas durante cinco días y la paciente se retira del lugar de atención odontológica en óptimas condiciones.

A la hora siguiente, la paciente regresa presentando leve sangrado nasal. Se inspecciona la cavidad bucal, la cual se encuentra en condiciones de normalidad, se inspecciona la zona nasal y se encuentra que el diente extraído estaba en comunicación oronasal, pues se observa una pequeña perforación en el piso de la cavidad nasal derecha de aproximadamente 3 $\mathrm{mm}$ de diámetro (Figuras $3 \mathrm{a}$ y $3 \mathrm{~b}$ ).

Se lava la cavidad nasal con solución salina, se seca la zona y se aplica presión por 10 minutos sobre el tejido nasal con ayuda de una pinza algodonera y un algodón envuelto en gasa; se decide no suturar, debido a que es una perforación pequeña y con la ayuda de un tapón anterior temporal se logra detener el sangrado (Figura 3c). La paciente se deja en observación por una hora, y se retira el tapón previamente humedecido con solución salina, para evitar retirar el coágulo que se ha formado.

Se continúa el seguimiento mediante contacto telefónico durante los primeros ocho días cada 24 horas y en los cuatro meses siguientes, cada semana; dentro de los controles la paciente refiere haber seguido todas las recomendaciones, refiere que ha presentado una evolución óptima.

A los cuatro meses, se cita a la paciente en la ciudad de Bucaramanga para hacer un seguimiento del caso, 
un control clínico y obtener una imagen radiográfica. Al examen clínico, se observa que ya no presenta elevación de la zona del paladar en el lado derecho, se evidencian tejidos en condiciones de normalidad, sin presencia de eritema, edema o inflamación; a la palpación, el tejido se encuentra firme, sin molestia a la presión y visualmente su aspecto es similar al compararlo con el lado opuesto (Figuras 4a y 4b). $\mathrm{Al}$ inspeccionar la cavidad nasal, se encuentra tejido nasal completamente continuo, sin perforaciones ni irregularidades en la superficie del tejido del piso de la cavidad nasal (Figura 4c). En la radiografía oclusal, se evidencia formación nueva de tejido óseo y ausencia de lesiones en la zona derecha, donde fue practicado el acto quirúrgico y se observa una imagen radiopaca compatible con trabeculado óseo (Figura 4d). La paciente refiere que no volvió a presentar ninguna complicación, dolor o molestia y que su proceso de recuperación fue totalmente satisfactorio.
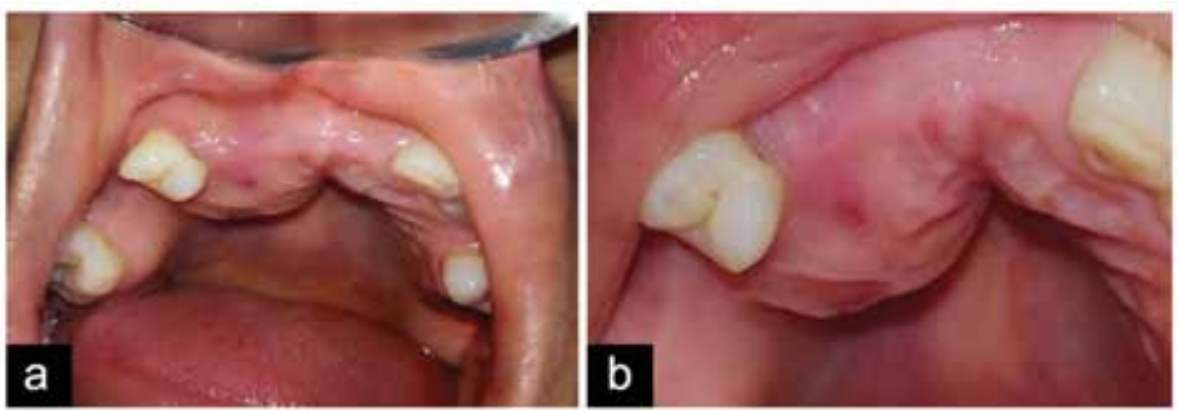

Figura 1. Hallazgos clínicos (a) Lesión en el paladar en la zona derecha de aproximandamente $25 \mathrm{~mm}$ de diámetro. (b) Punción realizada por el servicio de enfermería en la zona de la lesión.

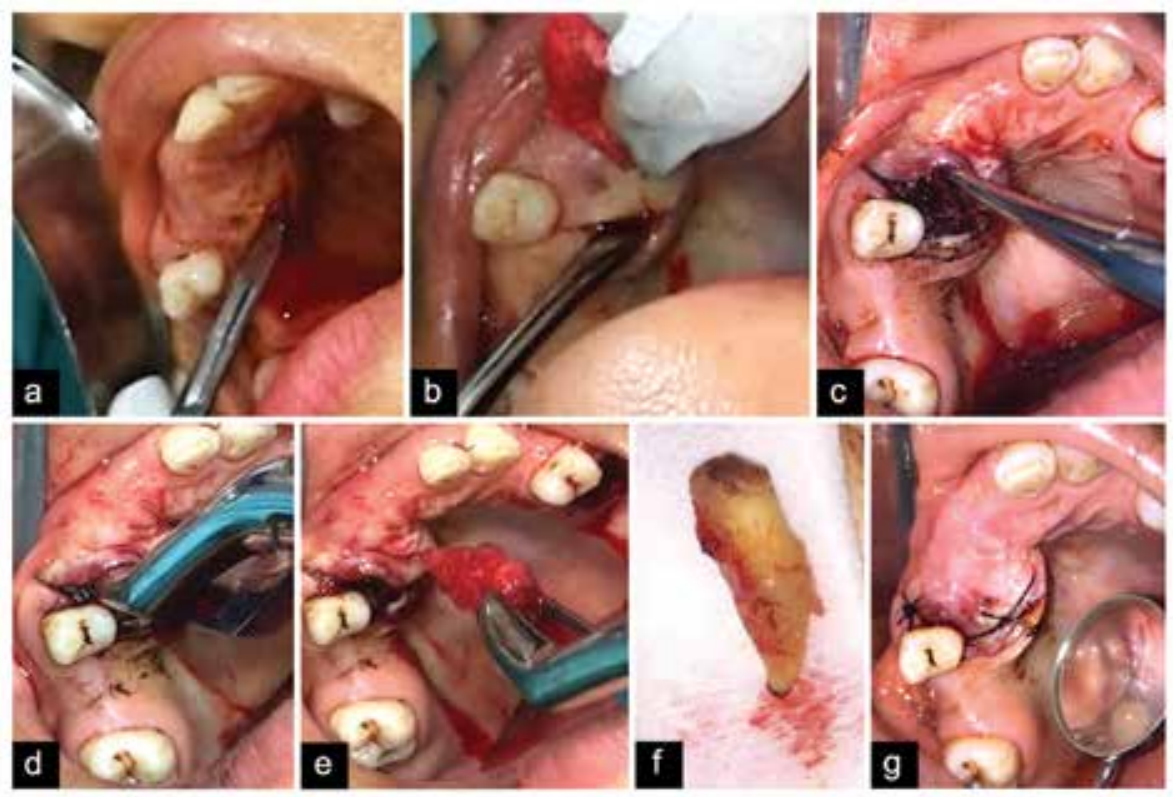

Figura 2. Abordaje quirúrgico (a) Incisión realizada con hoja de bisturí No. 12 sobre la lesión. (b) Inspección de la zona interna de la incisión con ayuda de un periostótomo de Molt. (c) Se realiza luxación del órgano dentario con ayuda de elevadores acanalados rectos. (d) Prehensión del órgano dental con el fórceps No. 65 lo más apical posible. (e) Exodoncia propiamente dicha del órgano dental en posición ectópica con ayuda de fórceps No. 65. (f) Órgano dental extraído sin corona y con presencia de zona de tonalidad oscura en la parte apical y tercio cervical compatible con necrosis pulpar. (g) Sutura del lugar de trabajo en forma de "X" en la zona del paladar y punto simple en la incisión relajante vestibular con sutura no reabsorbible 0,3 . 

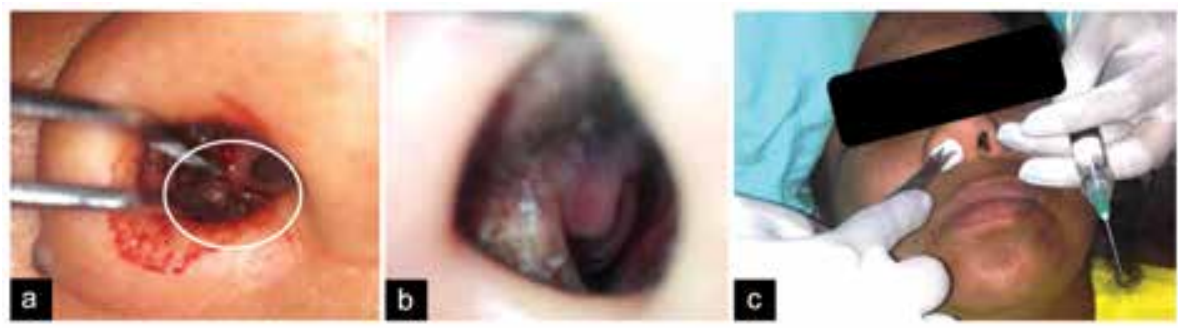

Figura 3. Abordaje post quirúrgico (a y b) Inspección de orificio en piso de cavidad nasal con ayuda de una pinza algodonera. (c) Lavado de la cavidad nasal derecha con solución salina, presión de la zona por diez minutos con algodón envuelto en gasa y tapón anterior temporal por una hora.

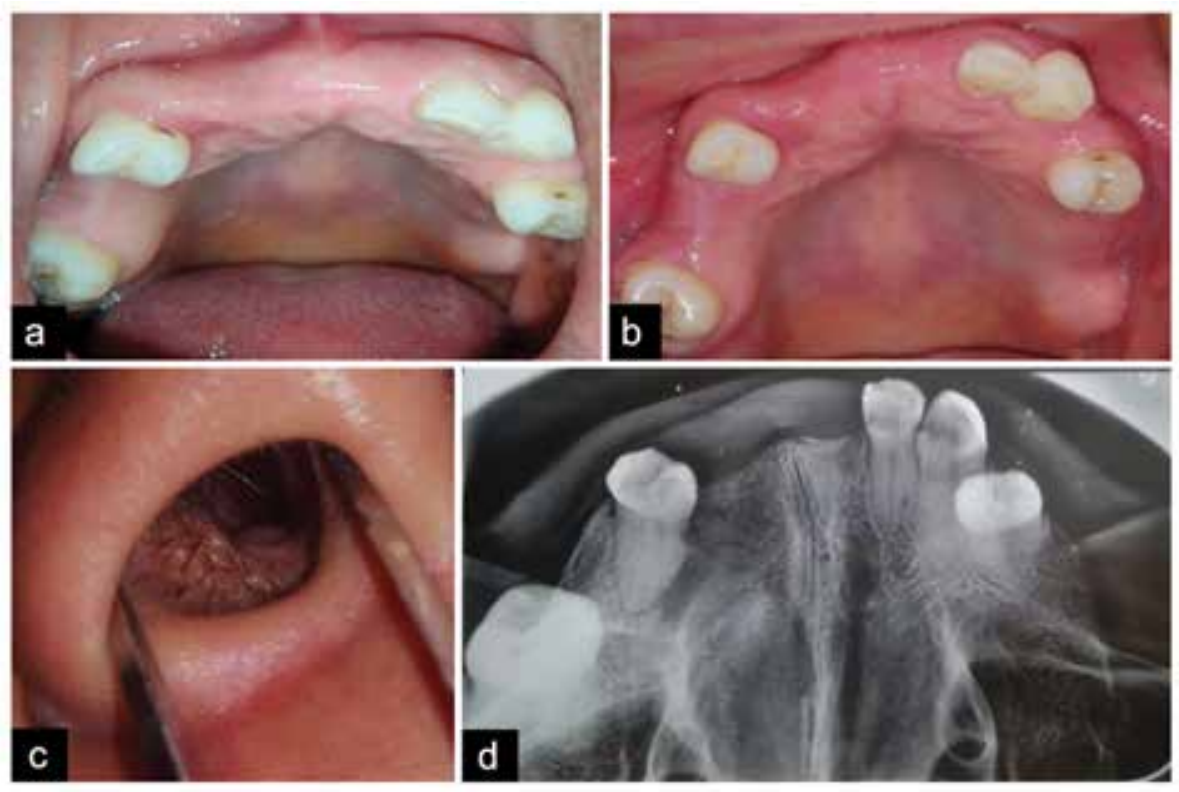

Figura 4. Control clínico y radiográfico luego de 4 meses (a y b) Paladar después de cuatro meses de la intervención quirúrgica, vista desde dos ángulos diferentes. (c) Piso de cavidad nasal en completa normalidad. Tejidos blandos de color rosado coral, continuos, sin perforación, ni alteración. (d) Radiografía oclusal en la que se evidencia zona radiopaca compatible con formación y continuidad de hueso palatino en la zona derecha.

\section{DISCUSIÓN}

Los dientes en posición ectópica son considerados como una patología de baja prevalencia, como consecuencia de un proceso de alteración en el patrón de desarrollo, posición, formación del germen dentario, traumas, entre otros ${ }^{4,7}$. La mayor parte de los órganos dentales en posición ectópica ubicados en el seno maxilar son los caninos superiores, seguidos de los terceros molares y los incisivos laterales superiores. Este tipo de casos se presenta con mayor frecuencia en mujeres, en un $66 \%$, con un promedio de edad 50 años ${ }^{8}$. Gran parte de los órganos dentales ectópi- cos retenidos en zonas cercanas del seno maxilar y cavidad nasal son asintomáticos, pero pueden llegar a presentar sinusitis, dolor crónico, pesadez a nivel facial, obstrucción nasal e inflamación del lado afectado de la cara ${ }^{8}$.

El tratamiento para seguir en estos casos, depende de la edad del paciente, la sintomatología, el tamaño de la pieza dentaría, la ubicación de esta, el espacio que presente en la arcada por tratar e incluso la reposición de la pieza dentaria con tratamiento ortodóntico y quirúrgico ${ }^{1,8,9}$. Aún con estas consideraciones, si se decide que la pieza dental se debe extraer, se 
sugiere que el órgano dental se encuentre completo o parcialmente erupcionado para disminuir las posibles complicaciones posteriores; aun más si la pieza dentaría está relacionada con un proceso infeccioso o un estado que comprometa la salud integral del paciente. Siempre se debe evaluar el riesgo/beneficio de la intervención y la relación con las estructuras anatómicas cercanas, considerando las posibles complicaciones y el pronóstico idóneo del tratamiento ${ }^{9}$. Sin embargo, autores como Cazar y col. argumentan que es preferible realizar la extracción desde un principio, fundamentado en las consideraciones anatómicas, para evitar que con el tiempo aparezcan quistes, tumores, infecciones y otras alteraciones ${ }^{8}$.

Algunas opciones quirúrgicas se pueden realizar a través de una vía endoscópica, la cual se puede tomar como primera opción para el correcto diagnóstico y tratamiento de cuerpos extraños encontrados dentro del seno maxilar, dependiendo del tamaño del órgano a extraer y el sangrado post endoscópico que se suele presentar ${ }^{4,3,8}$. Otras opciones incluyen hacer un abordaje transnasal, transpalatino, abordaje Cadwell-Luc o cuadrangular, el cual permite una mejor visión de las paredes internas en la zona de trabajo y finalmente se procede a realizar una reposición con materiales de osteosíntesis8. Al extirpar la pieza dentaria, se debe tener en cuenta que al finalizar la intervención se debe retirar todo el tejido blando que se encuentre rodeando el órgano dental retenido y lo ideal es tomar un estudio histopatológico de la pieza extraída y sus tejidos adyacentes para corroborar el diagnóstico.

En el caso actual, no fue posible identificar qué pieza era el órgano dental retenido, ya que este no tenía corona para identificarlo morfológicamente y ante la ausencia de los dientes adyacentes no fue posible deducir qué diente era, o si en su defecto era un diente supernumerario.

Los casos de dientes con erupción ectópica se suelen descubrir por medio de una radiografía al observar una imagen radiopaca compatible con tejido dental calcificado, rodeado en algunas ocasiones de una zona radiolúcida compatible con una lesión adyacente. Algunas de las ayudas radiográficas que se pueden utilizar para ubicar y reconocer la anatomía que rodea esta pieza dentaria en posición ectópica, son: Caldwell, Watters, lateral de cráneo, oclusal maxilar, ortopantomografía o panorámica y la ayuda radiográfica ideal que es la tomografía Cone-Beam, por producir imágenes de alta calidad y permitir una amplia visualización de la zona anatómica de interés ${ }^{4}$. Es recomendable involucrar al menos dos o más de estas ayudas radiográficas para contar con un panorama más claro de la intervención que se va a realizar y evaluar las estructuras anatómicas comprometidas con el fin de evitar al máximo los posibles riesgos, que pueden ser: desplazamiento de la pieza dentaría comprometiendo la vía digestiva o aérea y permitiendo que se creen infecciones recurrentes, o incluso la obstrucción del conducto nasolagrimal ${ }^{4}$.

Dentro de las limitaciones del caso tratado, está la ausencia de una radiografía inicial como ayuda diagnóstica, idealmente una tomografía Cone-beam y un estudio histopatológico posterior al tratamiento, pero debido a que la atención se brindó en un área rural y dentro de un servicio de salud extramural, fue imposible realizar este tipo de procedimiento; así mismo, se puede mencionar la falta de un rinoscopio para obtener mejores fotografías intranasales.

Una opción válida pero que no se realizó, era suturar los tejidos blandos de la cavidad nasal, sin embargo, dado que la perforación era muy pequeña, se logró controlar el sangrado por medio del tapón anterior temporal.

Así mismo, es pertinente mencionar las fortalezas de este reporte. Una de ellas, y quizás la más importante, es que finalmente se brindó atención odontológica a una persona de bajos recursos económicos procedente de un lugar remoto sin acceso a servicios de salud. Infortunadamente, esta situación es sumamente común en Colombia, especialmente dentro de las áreas rurales. Otra fortaleza para resaltar es el seguimiento continuo que se le hizo a la paciente, este seguimiento telefónico, clínico y radiográfico permitió evaluar la ausencia de posibles complicaciones y comprobar una satisfactoria evolución del tratamiento.

\section{CONCLUSIONES}

Se puede mencionar que la etiología de los dientes ectópicos es multifactorial y en algunos casos permanece desconocida. Los dientes que presentan mayor alteración en su línea eruptiva son los caninos 
superiores, los cuales terminan también impactados. Es importante estar alerta en caso de observar un retraso en su patrón de erupción, erupción asimétrica o ausencia de piezas dentales para ofrecer un plan de tratamiento oportuno y evitar alteraciones futuras.

\section{REFERENCIAS}

1. Trejo García W, Mendoza Rodríguez M, Medina Solís CE, Veras Hernández MA, Lucas Rincón SE, Casanova Rosado JF. Supernumerario invertido en paladar de un infante: Reporte de un caso clínico. Pediatría (Asunción). 2019;45(3):237-41.

2. Yaseen SM, Naik S, Uloopi KS. Ectopic eruption - A review and case report. Contemp Clin Dent. 2011;2(1):3-7. DOI: 10.4103/0976-237X.79289.

3. Fuentes Fernández R, Oporto Venegas G. Tercer molar ectópico impactado en zona retromolar. Reporte de Caso. Int J Morphol. 2009;27(1):35-8.

4. Liceaga-Escalera C, Vélez-Cruz M, Montoya-Pérez L, Torres-Urbina C. Erupción dental ectópica en el piso nasal. Reporte de tres casos. Rev Hosp Jua Mex. 2017;84(1):37-43.
5. Chen A, Huang J-K, Cheng S-J, Sheu C-Y. Nasal Teeth: Report of Three Cases. American Journal of Neuroradiology. 2002;23(4):671-3.

6. Öztürk C, Eryilmaz K, Çakur B. Supernumerary Tooth in the Nose. Turk J Med Sci. 2007;37(4):227-30. DOI: 10.1016/S0377-1237(08)80071-8.

7. Gutiérrez-Rojo JF, Gutiérrez-Villaseñor J, Mú-Gálvez BY, Navarrete Ayón KB, García-Rivera RN. Frecuencia de dientes con erupción ectópica de la Especialidad de Ortodoncia de la Universidad Autónoma de Nayarit. Revista Tamé. 2019;8(23):905-7.

8. Cazar Almache M, Palacios Vivar DE, Torres Cruz YJ, Miranda Villasana JE. Tercer molar ectópico en seno maxilar: reporte de un caso. Revista ADM. 2017;74(4):202-5.

9. Torres Madolnado C, Sibri Quizhpe C, Palacios Vivar D. Canino invertido en apófisis frontal del maxilar: reporte de un caso. Acta Odontol Colomb. 2018;8(1):75-82. DOI:10.15446/aoc.v8n1.70361.

\section{Correos electrónicos de los autores:}

Andrea del Pilar Albarracín-García: andrea_albarracin15@hotmail.com

Luisa Fernanda Muñoz: luisafm4@gmail.com

Gerardo Enrique Albarraín-García: gerardoal_7@hotmail.com 